\title{
I remember it now, so I'll remember it later: Working Memory Representations Guide Inaccurate Predictions of Future Memory Performance
}

\author{
Julia Krasnoff ${ }^{1} \&$ Alessandra Souza ${ }^{1,2}$ \\ University of Zurich ${ }^{1}$, University of Porto ${ }^{2}$
}

Note: This manuscript is a pre-print, and may not exactly replicate the final publication of this work.

Author Note

The project was funded by the Swiss National Science Foundation (grant number:

100014_179002). We thank Klaus Oberauer for helping with fitting the visual working memory confidence model.

Correspondence should be addressed to Julia Krasnoff, Department of Psychology, Cognitive Psychology Unit, University of Zurich, Binzmuehlestrasse 14/22, 8050 Zurich, Switzerland. E-mail: j.krasnoff@psychologie.uzh.ch. 


\begin{abstract}
Making accurate predictions of future memory performance (Judgements of Learning; JOLs) is a prerequisite for efficient learning. Since decades, those JOLs are assumed to be made inferentially, based on cues. This cue-utilization approach substituted the idea that JOLs are directly linked to memory quality. We criticize the reasons for the rejection of this memorystrength hypothesis because they ignore the existence of two different memory systems: working memory which holds representations immediately accessible, and long-term memory which is a more permanent store. Considering both memory systems, the current work revisited the memory-strength hypothesis: In Experiment 1, participants memorized sequences of two or four colored objects, then they provided JOLs for a long-term memory test, and performed a working memory test on the objects' colors. After learning 200 objects, the long-term memory test on all studied objects followed. Sequence-length affected working memory, but not long-term memory performance. JOLs, however, were higher for sequences of two than four and correlated higher with working memory than long-term memory performance. Experiment 2 replicated the sequence-length effect on JOLs in the absence of a working memory test. Results of a sequenceeight condition revealed an increase in JOLs' accuracy when the number of studied objects exceeded working memory span. Contrary to predominant theories, our findings suggest that JOLs are based on the quality of memory representations.
\end{abstract}




\section{Working Memory Representations Guide Inaccurate Predictions of Future Memory Performance}

A growing body of research has investigated how people make Judgements of Learning (JOLs): predictions about future retrieval of studied information. According to the predominant theory in this field, the cue-utilization approach proposed by Koriat (1997), people do not have direct access to their memory content but rely on a variety of cues to make inferences about the probability of a successful, delayed retrieval. Those cues can be intrinsic (e.g., item difficulty) or extrinsic (e.g., presentation duration) to the memoranda, and mnemonic (e.g., ease of retrieval). Because some cues are more predictive for future performance than others, JOLs vary in how well they predict performance.

The cue-utilization approach substituted the previously prevalent hypothesis that JOLs are based on memory strength (e.g., Cohen et al., 1991). Koriat (1997) discarded this memorystrength hypothesis arguing that it could not explain why some experimental manipulations affect JOLs but not delayed memory performance (and vice versa): If memory strength were the basis of both, JOLs and delayed performance, every manipulation affecting delayed performance should similarly affect JOLs. In contrast, studies showed that certain manipulations (e.g. item repetition) affect participants' JOLs and delayed performance in different ways (Koriat, 1997). These findings, and the general inaccuracy of JOLs (Bjork et al., 2013), were taken as evidence to reject the memory-strength hypothesis.

There are two crucial aspects that were overlooked by this argument. First, it ignores that JOLs and delayed performance are assessed at two different time-points. This only holds under the assumption that memory representations remain stable over time or, at least, degrade at a similar speed independent of the experimental manipulation. However, studies (e.g., Roediger \& 
Karpicke, 2006) have shown that some manipulations (e.g., repeated study) increase performance when memory is tested after short delays (e.g., $5 \mathrm{~min}$ ), but harm performance at longer delays (1-week). Conversely, other manipulations (e.g., testing memory) lead to worse performance at short delays, but increased performance at longer delays. Here, everyone would agree that performances at both time-points depend on memory strength, although they are uncorrelated.

The second and more germane critique is that this argument does not consider the involvement of two different memory systems, namely working memory (WM) which maintains a small amount of information accessible in mind, and long-term memory (LTM) which is a more permanent memory store. JOLs are commonly made immediately after studying. The study information is then still maintained in WM, while at delayed test information is retrieved from LTM. WM is capacity-limited (Cowan, 2010), and thus sensitive to manipulations, such as memory load, that do not influence LTM performance (e.g., Brady et al., 2013). Conversely, certain learning strategies (such as elaboration) were found to improve LTM but not WM (Bartsch \& Oberauer, 2021). This shows that WM and LTM representations are independent. We argue that it is necessary to consider both memory systems when examining whether the quality of memory representations is used as the basis for generating JOLs, and that neglecting the role of WM has led to discarding the memory-strength account too hastily.

Although the differentiation between WM and LTM is seldom made in the recent JOL literature, its relevance for JOLs has already been pointed out previously. Nelson and Dunlosky (1991) made this differentiation to explain the delayed-JOL effect: the observation that JOLs are more accurate when solicitated with a delay. They hypothesized that delayed JOLs are more accurate (than immediate JOLs) because both delayed JOLs and delayed performance are based 
on the strength of LTM representations. Conversely, immediate JOLs are less accurate because they are based on the strength of WM representations. More recent results are consistent with this so-called monitoring dual-memories hypothesis. The accuracy of immediate JOLs increases when they are preceded by a short distractor task (Bui et al., 2018), which disrupts WM maintenance. Contrarily, the accuracy of delayed JOLs decreases when the critical response (e.g., second word of word pair) is provided at JOL assessment (Dunlosky \& Nelson, 1992), which activates this representation in WM, obliterating LTM retrieval. Taken together, the delayed-JOL effect and other related findings can be explained by a memory-strength account, but only when the involvement of the two memory systems is considered.

The cue-utilization approach requires additional assumptions to explain the delayed-JOL effect or increased accuracy after distraction. Cue-utilization proponents argue that different cues are used in different time-points. Koriat (1997), for instance, suggested that people use less informative (intrinsic and extrinsic) cues when they are asked to give an immediate JOL, and that they use more informative, mnemonic cues when providing delayed JOLs. However, when exactly which cues are used is not explicitly described by the theory, which makes it hard to test.

Although, the idea that WM representations could influence JOLs is not novel, surprisingly, it has never been directly tested before. Across two preregistered experiments, we provide evidence that memory strength influences immediate JOLs by demonstrating that their inaccuracy reflects the usage of WM representations which are not always predictive of delayed performance. These results challenge the predominant view on how JOLs are made.

\section{Experiment 1}

In Experiment 1, we manipulated WM load when JOLs were requested to test whether WM representations are used to make immediate JOLs. Unlike most studies examining JOLs that 
used verbal material (for a meta-analysis see Double et al., 2018), we used a color-reproduction task which provides fine-grained information about memory quality. Brady et al. (2013) showed that memory load reduces WM fidelity; but not LTM fidelity. If people rely on WM representations to predict their future performance, JOLs should vary with WM load and this variation should be uncorrelated with LTM performance.

\section{Method}

Participants. We preregistered to collect a sample of 30 university students aged 18-35 years old. Our main manipulation consisted of the variation of memory load ( 2 vs. 4 objects in a sequence). A previous study from our lab using the same visual materials found a memory load effect of $d=2.09$ on immediate recall. To detect such a large effect with $\alpha=0.05$ and power $=$ $95 \%$, we would need a sample of only 5 participants. We decided to collect a sample-size six times larger because (a) we aimed to test whether the memory-load effect appears in the JOLs and delayed test, and (b) we initially aimed to fit the data with the Visual Working Memory Confidence model (van den Berg et al., 2017). Participants were recruited to take part in a labbased study in exchange for 15 CHF or partial course credit. They self-reported no visual impairment. The following criteria for the exclusion of participants were preregistered (https://osf.io/75dbw/?view_only=None): (a) Overall performance in the immediate recall task is not significantly different from chance level, (b) variance in JOLs is not statistically different from 0 and (c) the experiment is not completed. Based on these criteria no participants had to be excluded from the sample. 
Materials and Procedure. The experiment was programmed in MATLAB using the Psychophysics Toolbox 3 extension (Brainard, 1997). The main experimental task consisted of an object-color continuous reproduction task. Objects were sampled from 321 clip-arts obtained from the set used by Sutterer and Awh (2016). For each participant, 200 objects were randomly selected from this pool to serve as memoranda in the experimental trials. Additionally, six objects were selected for two practice trials. Each object was randomly assigned one of 360 colors selected from a continuous color wheel defined in the CIELAB color space with $L=70, a=20$, and $b=38$, and radius $=60$. The experimental procedure is depicted in Figure 1.

\section{Figure 1}

Experimental Procedure of Experiment 1.

\section{Learning Phase}

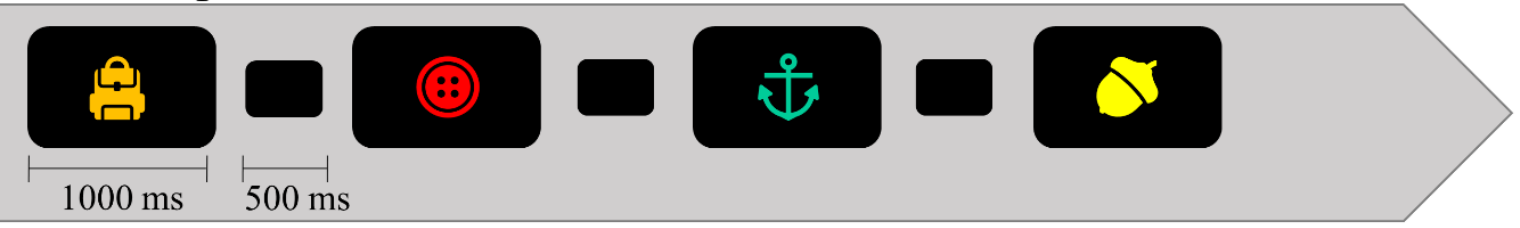

2. Judgements of Learning

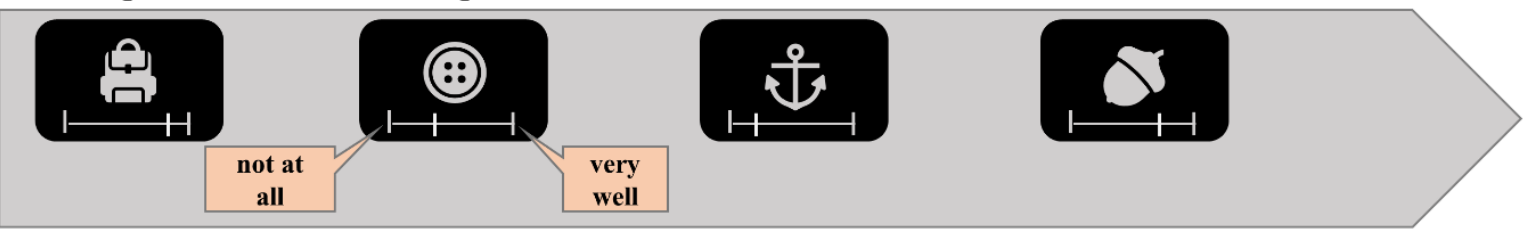

3. Immediate Test

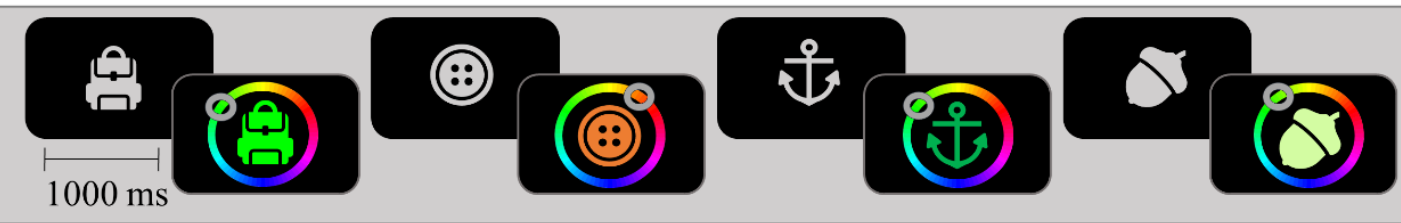

4. Delayed Test (of all studied objects)
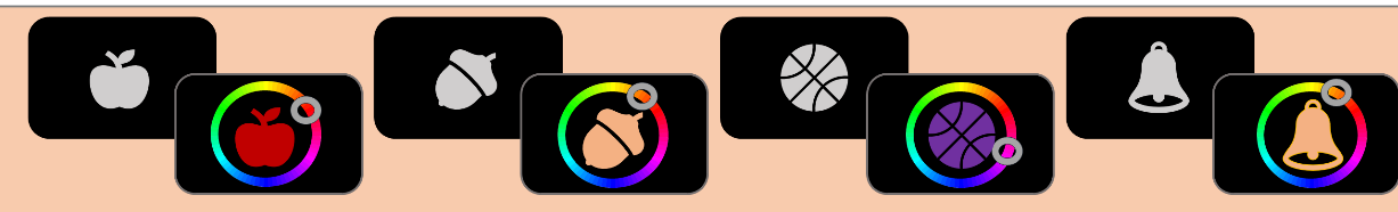

Note. Flow of events of Experiment 1. Step 1-3 depict a trial with a sequence-length of four.

These steps repeated several times with varying sequence-lengths. Step 4 was conducted only once after all sequences have been presented. 
Participants started the learning phase with a mouse-click. During the learning phase, a sequence of either two (low WM load) or four (high WM load) colored objects was presented for

study. Each colored object was presented on the screen center (objects' size $=100$ pixels) against a black background for $1 \mathrm{~s}$. Object offset was followed by a 0.5 blank inter-stimulus interval before the next colored object was presented. Following each sequence of two or four objects, JOLs were solicited. The silhouette of each object was presented in light grey with a scale ranging from 1 to 100 presented below it. The objects appeared in the same order as they were presented in the learning phase. Participants rated how well they thought they will remember the object's color in a delayed test in the very end of the experiment. Next, an immediate (WM) test followed. In the original sequence order, the silhouette of each object of the sequence appeared again on the screen center, and $1 \mathrm{~s}$ later a color wheel surrounded it. Participants used the mouse to select the color they remembered for the depicted object from the color wheel. When moving the mouse cursor along the wheel, the object was filled with the color at the current mouse position. When participants were satisfied with the selected color, they pressed the mouse button to confirm their response. Altogether, 100 objects were presented in each sequence-length condition (namely 50 trials of sequence-length two and 25 trials of sequence-length four). Finally, at the end of the experiment, participants performed a delayed (LTM) test where they reproduced the color of each studied object in random order. The test procedure was the same as for the immediate recall task.

\section{Results}

\section{Sequence-length effect on recall and judgements of learning.}

We computed a measure of recall error for the immediate and the delayed test by computing the absolute difference between the presented color and participants' response. This 
measure can range from $0^{\circ}$ (the correct color was chosen) to $180^{\circ}$ (a color on the opposite side of the color wheel was chosen). We preregistered and performed a Bayesian ANOVA with sequence-length and test time-point as predictors, participants as a random effect, and recall error as predicted variable. The results provided strong evidence in favor of an interaction between sequence-length and test time-point on recall error, $\mathrm{BF}_{10}=262.43$. We then performed two additional Bayesian $t$-tests for paired-samples to investigate the influence of sequence-length on recall error separately for the immediate, and delayed test. As depicted in Figure 2A, immediate recall error was lower for objects presented in sequences of two than four, $\mathrm{BF}_{10}=7.87 \times 10^{5}$. As expected, this sequence-length effect was not found for the delayed test (see Figure $2 \mathrm{~B}$ ), $\mathrm{BF}_{01}=$ 4.51. Even tough delayed performance was not influenced by sequence-length, participants gave - as predicted - higher JOLs to objects learned in sequences with two compared to four objects (see Figure $2 \mathrm{C}), \mathrm{BF}_{10}=19.78$. 


\section{Figure 2}

Recall Error in the Immediate and Delayed Test and Judgements of Learning as a Function of Sequence-Length.
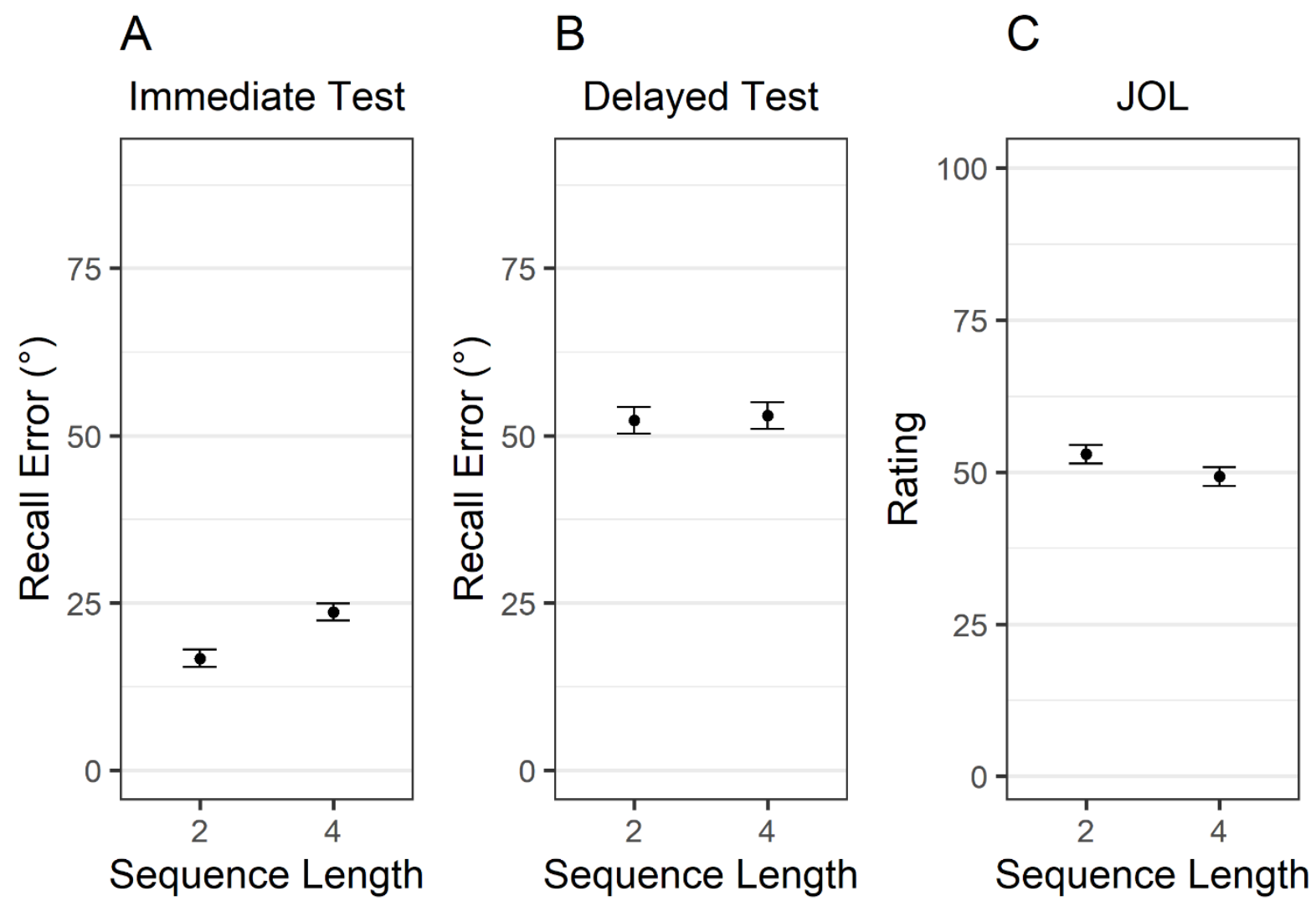

Note. Error bars represent 95\% within-participant confidence intervals.

\section{Correlations between immediate performance, delayed performance, and}

judgements of learning.

For each participant, a correlation between JOLs and recall error in the delayed test was computed following our preregistered analysis plan. This correlation reflects the accuracy of their JOLs. Additionally, we computed the correlation between recall error in the immediate test and JOLs, reflecting how strongly JOLs are related to the quality of WM representations.

Because recall error and JOLs were not normally distributed in our sample, we also computed the Spearman correlation coefficient. Both, Pearson's and Spearman's correlation coefficients are 
depicted in Figure 3. The correlations were transformed to Fishers $z$-scale before computing the average or performing Bayesian $t$-tests.

\section{Figure 3}

Pearson and Spearman Correlations between Judgements of Learning and Recall Error in the Delayed Test (LTM) and Immediate Test (WM).
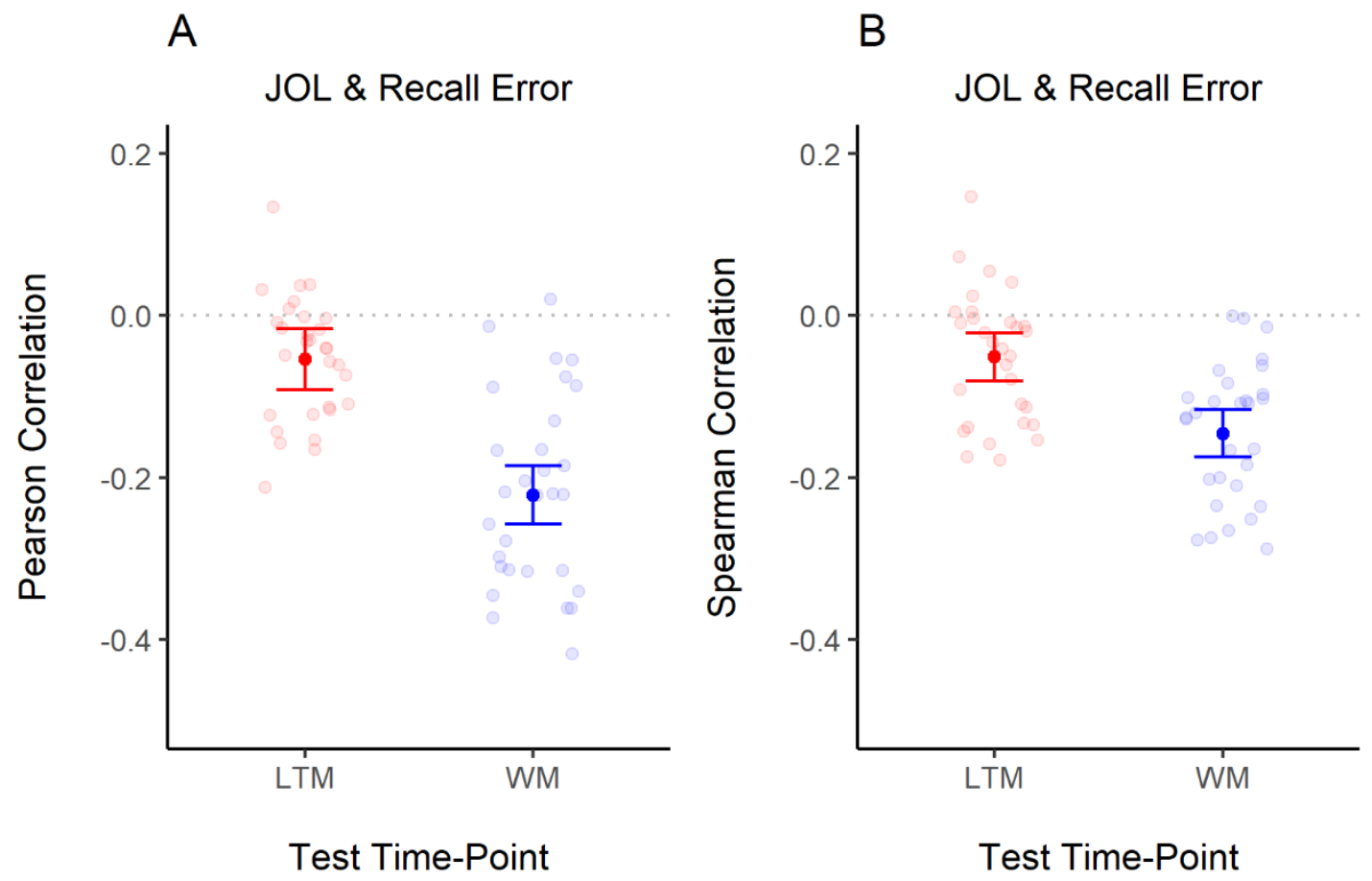

Note. Error bars represent $95 \%$ within-participant confidence intervals. Transparent dots represent correlation coefficients of individual participants. Correlation coefficients were transformed to Fisher's $z$-scale prior to averaging them and computing confidence intervals. They were then retransformed to the $r$-scale.

Despite reflecting people's predictions for delayed memory performance, JOLs correlated higher with immediate than with delayed recall error $\left(\mathrm{BF}_{10}=5.25 \times 10^{4}\right.$ using Pearson correlation, $\mathrm{BF}_{10}=373$ using Spearman correlation). 
As preregistered, we implemented the confidence model proposed by van den Berg and colleagues (2017) and fitted it to our data to examine whether it can predict participants' JOLs, but we did not obtain stable estimates (see Supplementary Materials File, https://osf.io/pqm63/?view_only=9c66760d353448cb8e300a43379af988).

\section{Discussion}

Experiment 1 provided first evidence for the use of WM representations when making immediate JOLs. Although sequence-length did not affect delayed performance, participants gave higher JOLs in sequences of two than four, and JOLs were more correlated with immediate than delayed performance.

One alternative explanation, however, is that the inclusion of the immediate test artificially increased the salience of WM representations, therefore "nudging" participants into using them as a basis for JOLs. This possible limitation was addressed in Experiment 2.

\section{Experiment 2}

In Experiment 2, we examined whether WM representations are intuitively used to make JOLs by introducing a between-subject condition to the design of Experiment 1: While some participants made JOLs and then engaged in an immediate test, other participants solely made JOLs during the study phase. If JOLs also vary as a function of sequence-length in a "no-test" condition, this would provide clear evidence for the intuitive use of WM representation as a basis for JOLs.

Another goal of Experiment 2 was to test whether JOL accuracy increases when information is retrieved from LTM. To attain this, we included a sequence-length eight condition, which exceeds WM capacity hence requiring participants to retrieve information from LTM. We 
expect the correlation between immediate and delayed performance to be higher when eight objects are studied in one sequence (as compared to two and four). Consequently, JOL accuracy should also be higher in this condition. Inclusion of this sequence-length eight condition could thus provide additional insights into why delayed JOLs are more accurate: after a delay, WM representations are replaced and retrieval from LTM is enforced.

\section{Method}

Participants. We preregistered to start data collection with a sample $N=60$, because we included a between subject manipulation. Given our reliance on Bayesian inferences, we planned to increase sample size if we obtained ambiguous evidence $(\mathrm{BF}<3)$ to reject or accept our hypotheses. Accordingly, 60 participants aged 18 to 35 years, without visual impairment were recruited on the platform Prolific to take part in an online experiment for 6.25 GBP. Only English native speakers were recruited, to reassure the understanding of instructions. No participant had to be excluded based on the same preregistered exclusion criteria as were applied in Experiment 1 (see https://osf.io/j78un/?view_only=None).

The design of the experiment consisted of a between-subjects manipulation of immediate memory test (test vs. no-test) and a within-subjects manipulation of sequence-length $(2,4$, or 8$)$. Participants were randomly assigned to one of the two between-subject conditions resulting in 41 participants in the test condition and 19 participants in the no-test condition. This sample size was sufficient to obtain substantial evidence in favor or against our hypothesis, and hence we stopped data-collection. 
Materials and Procedure. Due to the COVID-19 restrictions, the experiment was conducted online. The task was programmed using lab.js (Henninger et al., 2019). We created a pool of 209 concrete objects. For each participant, 192 objects were randomly selected to serve as memoranda in the experimental trials. Additionally, four objects were selected to serve for one practice trial. Overall, the experiment consisted of 32 trials of length two, 16 trials of length four, and 8 trials of length eight. As before, each object was randomly assigned one of 360 colors selected from a continuous color wheel defined in the CIELAB color space with $L=70, a=20$, and $\mathrm{b}=38$, and radius $=60$ (Zhang \& Luck, 2008).

As in Experiment 1 (see Figure 1), participants completed cycles consisting of a (1) learning phase followed by a (2) JOL phase and - in case they were in the "immediate test" condition - (3) an immediate test phase. The experiment ended again with a delayed LTM test of all 192 objects. A minor change to the procedure of Experiment 1 was that at the immediate and delayed test, the color wheel appeared directly together with the silhouette of the objects. Apart from this and the inclusion of a "sequence-length eight" condition and a "no immediate test" condition, the experimental procedure was kept the same as in Experiment 1.

\section{Results}

\section{Sequence-length effect on recall and judgements of learning.}

Figure 4 shows the average recall error as function of sequence-length, test time-point and test condition. As in Experiment 1, we preregistered and performed a Bayesian ANOVA with sequence-length and test time-point as predictors, participant as a random effect, and recall error as dependent variable. For this analysis, we only used data of participants from whom we had observations for the immediate and delayed test (i.e., participants in the test condition). We found evidence for an interaction between sequence-length and test time-point on recall error (see 
Figure 4A, Figure 4B), $\mathrm{BF}_{10}=4.61 \times 10^{53}$, replicating the results of Experiment 1. A Bayesian hierarchical analysis yielded the same results (see Appendix A).

\section{Figure 4}

Recall Error in Immediate and Delayed Test and JOLs as a Function of Sequence-Length and Test Condition in Experiment 2.

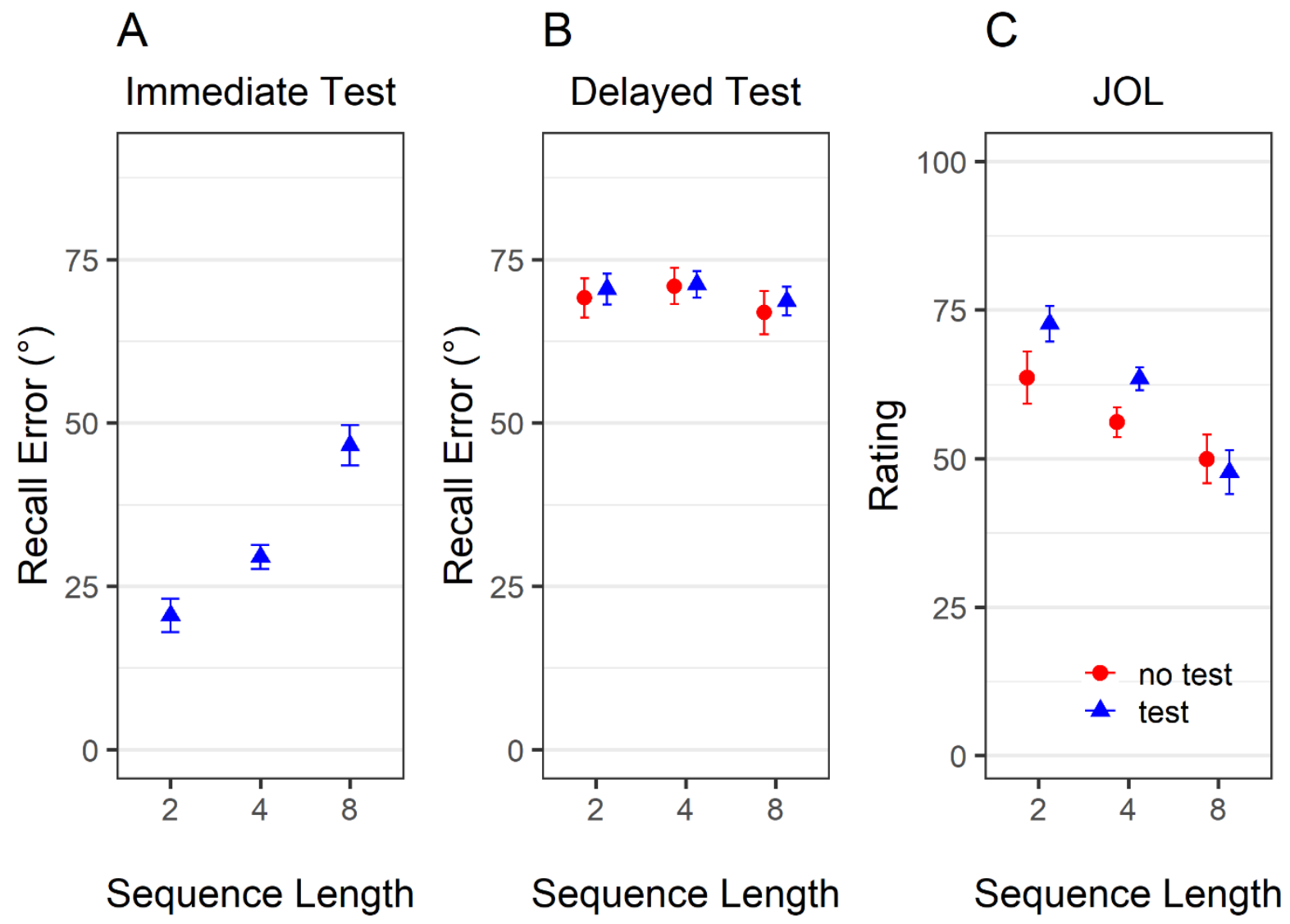

Note. Error bars represent 95\% within-participant confidence intervals.

In the next step, we conducted the preregistered analysis of participants' JOLs as a function of sequence-length and test condition (see Figure 4C). The Bayesian ANOVA provided substantial evidence for an interaction between sequence-length and test condition on $\mathrm{JOLs}_{\mathrm{B}} \mathrm{BF}_{10}$ $=9.13 \times 10^{14}$. Thus, sequence-length affected JOLs differently depending on whether participants performed the immediate test or not. The same result was obtained using a Bayesian hierarchical analysis (see Appendix B). Critically, our hypothesis was that sequence-length affected JOL even 
in the absence of a WM test. To test this prediction, we computed another Bayesian ANOVA, this time only for the participants in the no-test condition. Here again we found overwhelming evidence for an effect of sequence-length on JOLs, $\mathrm{BF}_{10}=7.17 \times 10^{24}$ : JOLs were influenced by sequence-length irrespective of the immediate test, although sequence-length did not affect delayed performance.

\section{Correlations between immediate performance, delayed performance, and} judgements of learning.

As in Experiment 1, we computed correlations between JOLs and the recall error in the immediate and delayed test, respectively. Again, JOLs were more correlated with immediate than delayed performance (see Figure 5). Bayesian $t$-tests provided evidence for this difference, $\mathrm{BF}_{10}$ $=1.64 \times 10^{9}$ (for Spearman correlations) and $\mathrm{BF}_{10}=1.23 \times 10^{10}$ (for Pearson correlations). 


\section{Figure 5}

Pearson and Spearman Correlations between Judgements of Learning and Recall Error in the Delayed Test (LTM) and Immediate Test (WM) of Experiment 2.

A

JOL \& Recall Error

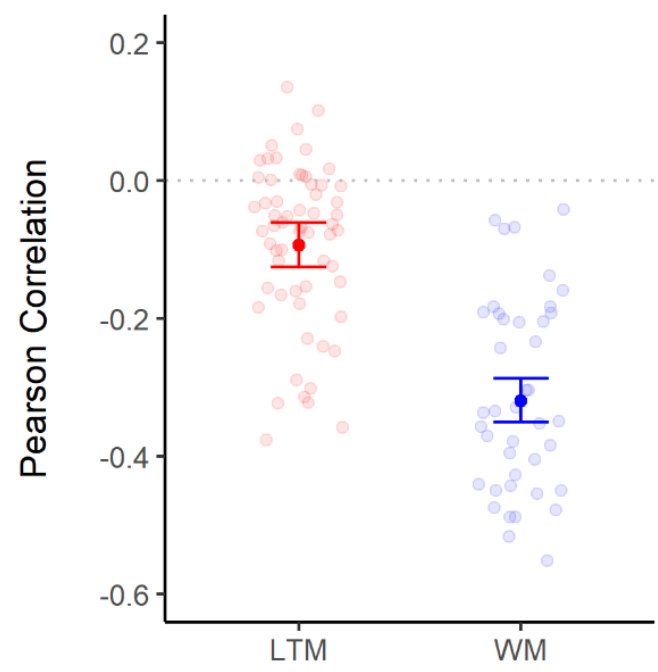

Test Time-Point
B JOL \& Recall Error

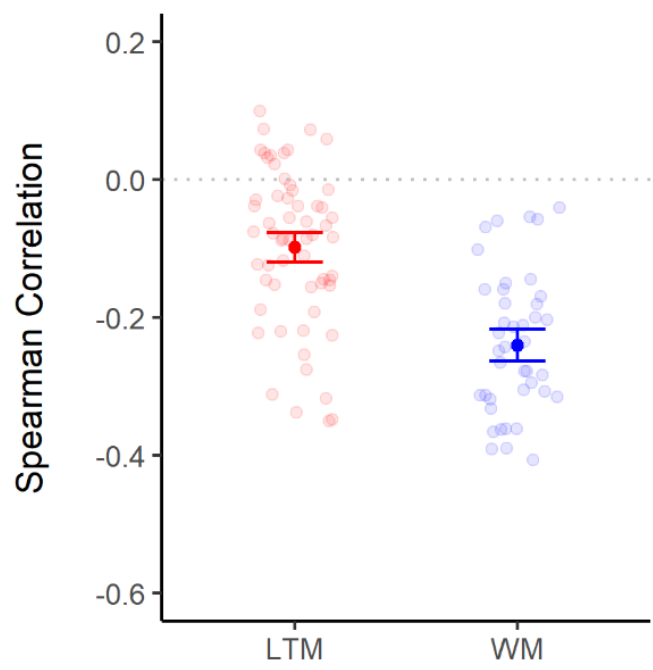

Test Time-Point

Note. Error bars represent $95 \%$ between-participant confidence intervals. Transparent dots represent correlation coefficients of individual participants. Correlation coefficients were transformed to Fisher's $z$-scale prior to averaging them and computing confidence intervals. They were then retransformed to the $r$-scale.

We then computed the correlation between immediate and delayed recall error, JOLs and delayed recall error (JOLs' accuracy) and JOLs and immediate recall error for each sequencelength condition (see Figure 6). 


\section{Figure 6}

Correlation between Immediate and Delayed Recall Error, JOLs and Delayed Recall Error and JOLs and Immediate Recall Error as a Function of Sequence-Length and Type of Correlation in Experiment 2.
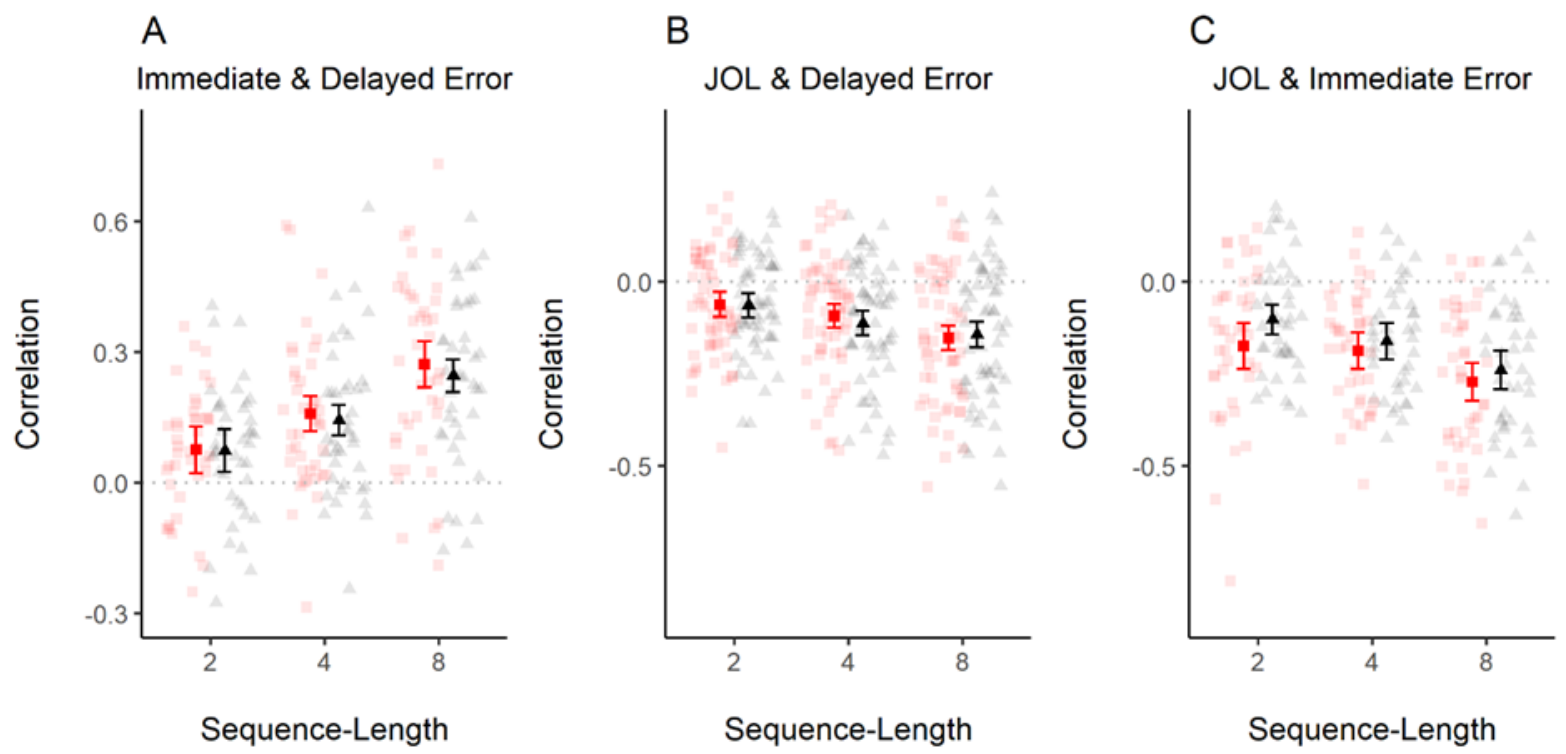

Note. Red squares depict Pearson correlation coefficients. Black triangles depict Spearman correlation coefficients. Error bars represent 95\% within-participant confidence intervals. Correlation coefficients were transformed to Fisher's $z$-scale prior to averaging them and computing confidence intervals. They were then retransformed to the $r$-scale.

Our prediction was that the correlation between immediate and delayed recall increases with increasing sequence-length. This prediction bases on the assumption that participants have to retrieve the color from LTM in both, the immediate and delayed test, when number of colored objects exceeds their WM span. In contrast, when the number of colored objects is within participants' WM span, they retrieve the colors from WM (when engaging in an immediate test or making JOLs presumably) but have to retrieve the colors from LTM when engaging in the 
delayed test leading to less accurate JOLs. Consistent with our prediction, the correlation between immediate and delayed performance was highest in the sequence-length eight condition (see Figure 6A). Our preregistered Bayesian modelling analysis led to the same result (see Appendix C).

In the last step, we examined the correlation between JOLs and delayed recall error, participants' JOLs' accuracy (see Figure 6B). As predicted, JOLs' accuracy varied with sequence-length: Participants were most accurate in predicting their future performance in the sequence-length eight condition, which exceeds average WM span.

Lastly, we observed correlations between JOLs and immediate recall error in all sequence-length conditions (Figure 6C), but these correlations were higher with increasing sequence-length. It is important to note that immediate recall error depends on the sequencelength condition: it is smallest in the sequence-length two and biggest in sequence-length eight condition. By analyzing the data of each sequence-length separately, we artificially restrict the variance of the immediate recall error. Consequently, the correlation coefficients that are computed based on the immediate recall data of individual sequence-length conditions are decreased.

\section{Discussion}

Experiment 2 corroborates the findings of Experiment 1: People intuitively use the quality of their current memory representations to make JOLs, even in the absence of an immediate test. Additionally, we observed an increase in JOLs' accuracy in the sequence-length eight condition. This increase can be explained by the involvement of LTM when WM span is exceeded. 


\section{General Discussion}

By considering the involvement of WM and LTM, we provided first evidence for the memory-strength account for JOLs. When JOLs are made immediately after studying, and the amount of information is within WM span, the quality of WM representations is used to predict future performance. In contrast, when WM span is exceeded, JOLs are also based on the quality of LTM representations. Because LTM representations also guide performance in the delayed test, JOLs' accuracy increases in the latter case. Our findings are therefore in line with the monitoring dual-memories explanation for the delayed-JOL effect.

One could now oppose that the sequence-length effect on JOLs is not related to the use of memory strength but just a reflection of the use of sequence-length as an extrinsic cue. This explanation, however, fails to account for two findings. First, it cannot explain why JOLs' accuracy increases for the sequence-length eight. Second, it fails to account for the correlation between immediate recall error and JOL that was found within each sequence-length condition and that is thus independent of our manipulation.

It is critical to note that recall error and JOLs were not perfectly correlated in our experiments. One explanation for this is that recall error is not a pure measure of the quality of memory representations but measures it with certain measurement noise. This alone, however, is not enough to account for the moderate correlations that we found. The strength of the correlations more likely implies that there are other factors (e.g., beliefs about relation of current and future performance, metacognitive ability) besides memory strength that influence JOLs but that remained unidentified in the present work.

Whereas our findings support the memory-strength account for JOLs, they do not generally discard the use of cues. Further research is needed to understand how these findings fit 
into the JOL literature of the last decades that offered strong support for the cue-utilization approach (Rhodes \& Castel, 2008; Tauber \& Rhodes, 2010; Undorf \& Erdfelder, 2011). At present, we can only consider possible reasons why the use of cues could have been overestimated in the past. One consideration is that variables that were considered as intrinsic or extrinsic cues may have affected the quality of WM representations. For instance, the finding that presentation duration influences JOLs could support both, the cue-utilization, and the memory-strength approach: Either people could have used the duration as an extrinsic cue for JOLs, or the duration could have affected the quality of WM representations given that there is ample evidence that presentation rate affects WM performance (Souza \& Oberauer, 2018; Tan \& Ward, 2008). Because the quality of memory representations at the assessment of JOLs has not been measured in the recent literature, these findings were directly interpreted as evidence for the cue-utilization approach. A similar concern can be raised regarding the evidence for the use of mnemonic cues, such as ease of retrieval (Koriat \& Ma'ayan, 2005). Retrieval ease is commonly measured by retrieval success or latencies. These measures, however, are highly correlated with memory strength. The finding that they predict JOLs could therefore just reflect the use of memory strength in contrast to posing clear evidence for the use of mnemonic cues.

Another consideration is that most studies requested JOLs in situations in which memory quality has low variation. Often items are sequentially presented and JOLs are assessed directly after their offset (e.g., Mueller et al., 2014), hence the to-be-rated items are perfectly maintained in WM when JOLs are assessed. The lack of memory quality variance paired with the requirement to give different JOLs to different items could have nudged participants into using rather irrelevant cues, such as font size (Rhodes \& Castel, 2008), when making JOLs. This is also consistent with our finding, that the association of immediate recall error with JOLs is 
weaker in conditions with consistently high performance (sequence-length two), whereas it is higher when performance varies more (sequence-length eight). Future research should explore whether people still use those cues when having more valuable information, such as a variation in the quality of memory representations.

Taken together, this work provides evidence for the long-rejected memory-strength hypothesis and challenges the predominant view of exclusively inferential, cue-based JOLs. When asked to make a prediction about future retrieval success, people use the quality of their current memory representations - even if it is sometimes misleading.

\section{Open Practices Statement}

The data and analyses scripts of both experiments are available via the Open Science Framework (https://osf.io/47wjp/?view only=9c66760d353448cb8e300a43379af988). The study material is available on request from the corresponding author. Both experiments were preregistered. 


\section{References}

Bartsch, L. M., \& Oberauer, K. (2021). The effects of elaboration on working memory and longterm memory across age. Journal of Memory and Language, 118, 104215. https://doi.org/10.1016/j.jml.2020.104215

Bjork, R. A., Dunlosky, J., \& Kornell, N. (2013). Self-regulated learning: Beliefs, techniques, and illusions. Annual Review of Psychology, 64, 417-444. https://doi.org/10.1146/annurevpsych-113011-143823

Brady, T. F., Konkle, T., Gill, J., Oliva, A., \& Alvarez, G. A. (2013). Visual Long-Term Memory Has the Same Limit on Fidelity as Visual Working Memory. Psychological Science, 24(6), 981-990. https://doi.org/10.1177/0956797612465439

Brainard, D. H. (1997). The Psychophysics Toolbox. Spatial Vision, 10(4), 433-436. https://doi.org/10.1163/156856897X00357

Bui, Y., Pyc, M. A., \& Bailey, H. (2018). When people's judgments of learning (JOLs) are extremely accurate at predicting subsequent recall: the "Displaced-JOL effect." Memory, 26(6), 771-783. https://doi.org/10.1080/09658211.2017.1406523

Bürkner, P. C. (2017). brms: An R package for Bayesian multilevel models using Stan. Journal of Statistical Software, 80(1). https://doi.org/10.18637/jss.v080.i01

Bürkner, P. C. (2018). Advanced Bayesian multilevel modeling with the R package brms. $R$ Journal, 10(1), 395-411. https://doi.org/10.32614/rj-2018-017

Cohen, R. L., Sandler, S. P., \& Keglevich, L. (1991). The failure of memory monitoring in a free recall task. Canadian Journal of Psychology/Revue Canadienne de Psychologie, 45(4), 523-538. https://doi.org/10.1037/h0084303

Cowan, N. (2010). The magical mystery four: How is working memory capacity limited, and 
why? Current Directions in Psychological Science, 19(1), 51-57.

https://doi.org/10.1177/0963721409359277

Double, K. S., Birney, D. P., \& Walker, S. A. (2018). A meta-analysis and systematic review of reactivity to judgements of learning. Memory, 26(6), 741-750.

https://doi.org/10.1080/09658211.2017.1404111

Dunlosky, J., \& Nelson, T. O. (1992). Importance of the kind of cue for judgments of learning (JOL) and the delayed-JOL effect. Memory \& Cognition, 20(4), 374-380. https://doi.org/10.3758/BF03210921

Henninger, F., Shevchenko, Y., Mertens, U. K., Kieslich, P., \& Hilbig, B. (2019). lab.js: A free, open, online study builder. 6. https://doi.org/10.31234/osf.io/fqr49

Koriat, A. (1997). Monitoring One’s Own Knowledge during Study: A Cue-Utilization Approach to Judgments of Learning. Journal of Experimental Psychology: General, 126(4), 349-370. https://doi.org/10.1037/0096-3445.126.4.349

Koriat, A., \& Ma'ayan, H. (2005). The effects of encoding fluency and retrieval fluency on judgments of learning. Journal of Memory and Language, 52(4), 478-492. https://doi.org/10.1016/j.jml.2005.01.001

Mueller, M. L., Dunlosky, J., Tauber, S. K., \& Rhodes, M. G. (2014). The font-size effect on judgments of learning: Does it exemplify fluency effects or reflect people's beliefs about memory? Journal of Memory and Language, 70(1), 1-12.

https://doi.org/10.1016/j.jml.2013.09.007

Nelson, T. O., \& Dunlosky, J. (1991). When People’s Judgments of Learning (JOLs) are Extremely Accurate at Predicting Subsequent Recall: The “delayed-JOL effect." Psychological Science, 2(4), 267-270. https://doi.org/10.1111/j.1467-9280.1991.tb00147.x 
Rhodes, M. G., \& Castel, A. D. (2008). Memory Predictions Are Influenced by Perceptual Information: Evidence for Metacognitive Illusions. Journal of Experimental Psychology: General, 137(4), 615-625. https://doi.org/10.1037/a0013684

Roediger, H. L., \& Karpicke, J. D. (2006). Test-enhanced learning: Taking memory tests improves long-term retention. Psychological Science, 17(3), 249-255. https://doi.org/10.1111/j.1467-9280.2006.01693.x

Souza, A. S., \& Oberauer, K. (2018). Does articulatory rehearsal help immediate serial recall? Cognitive Psychology, 107(September), 1-21. https://doi.org/10.1016/j.cogpsych.2018.09.002

Sutterer, D. W., \& Awh, E. (2016). Retrieval practice enhances the accessibility but not the quality of memory. Psychonomic Bulletin and Review, 23(3), 831-841. https://doi.org/10.3758/s13423-015-0937-x

Tan, L., \& Ward, G. (2008). Rehearsal in immediate serial recall. Psychonomic Bulletin and Review, 15(3), 535-542. https://doi.org/10.3758/PBR.15.3.535

Tauber, S. K., \& Rhodes, M. G. (2010). Does the amount of material to be remembered influence judgements of learning (JOLs)? Memory, 18(3), 351-362. https://doi.org/10.1080/09658211003662755

Undorf, M., \& Erdfelder, E. (2011). Judgments of Learning Reflect Encoding Fluency: Conclusive Evidence for the Ease-of-Processing Hypothesis. Journal of Experimental Psychology: Learning Memory and Cognition, 37(5), 1264-1269. https://doi.org/10.1037/a0023719

van den Berg, R., Yoo, A. H., \& Ma, W. J. (2017). Fechner's law in metacognition: A quantitative model of visual working memory confidence. Psychological Review, 124(2), 197-214. 
https://doi.org/10.1037/rev0000060

Zhang, W., \& Luck, S. J. (2008). Discrete fixed-resolution representations in visual working memory. Nature, 453(7192), 233-235. https://doi.org/10.1038/nature06860 


\section{Appendix A}

Bayesian Model Predicting Test Performance (Kappa) by Sequence-Length and Test

Time-Point

We estimated a Bayesian Model using the R-package brms (Bürkner, 2017, 2018). In this model we predicted test performance with the continuous predictor sequence-length (centered on length 2) and the factor test time-point having as a reference the WM condition. Because the distribution of recall error is skewed, we did not model the recall error directly, but we modelled the deviance of the given response from the correct color. The deviance distribution is best described by a von Mises (normal distribution for circular space). As most responses are close to the correct response, this distribution peaks at a deviance of zero. The width of the von Mises distribution is captured by the precision parameter, Kappa. In our model we predicted Kappa by the main effects of sequence-length and test time-point and their interaction. Originally, we planned to allow for a non-linear relation of sequence-length and deviance, but non-linear parameters were not allowed for a von-mises family; hence we fitted a standard linear model with 4 chains, each with 2000 iterations (1000 iterations were discarded as burn-in). Parameters converged as indicated by the R-hat values below 1.05. Table A1 summarizes the results.

\section{Table A1}

Population-Level Estimates for the Effects of Sequence-Length, Test Time Point, and Their Interaction on Memory Precision (Kappa). Values are reported on a logarithmic scale.

\begin{tabular}{lcccc}
\hline & $\begin{array}{c}\text { Posterior } \\
\text { Estimate }\end{array}$ & $\begin{array}{c}\text { Posterior } \\
\text { Estimate } \\
\text { Error }\end{array}$ & $\begin{array}{c}\text { lower 95\% } \\
\text { CI }\end{array}$ & $\begin{array}{c}\text { upper 95\% } \\
\text { CI }\end{array}$ \\
\hline Sequence Length & 0.02 & 0.02 & -0.02 & 0.06 \\
Test Time Point & $\mathbf{2 . 4 9}$ & $\mathbf{0 . 1 6}$ & $\mathbf{2 . 1 6}$ & $\mathbf{2 . 8 1}$ \\
$\begin{array}{l}\text { Sequence } \\
\begin{array}{l}\text { Length*Test Time } \\
\text { Point }\end{array}\end{array}$ & $\mathbf{- 0 . 2 4}$ & $\mathbf{0 . 0 2}$ & $\mathbf{- 0 . 2 9}$ & $\mathbf{- 0 . 2}$ \\
\hline
\end{tabular}


Note. $\mathrm{CI}=$ credible interval. Credible parameter values (i.e., for which 0 is not included in the credible interval) are presented in bold.

The results support an interaction of test time-point and sequence-length on Kappa.

Whereas test time-point had a credible effect on Kappa, we did not find a main effect of sequence-length. The supplementary materials file presents the model's prediction against the data (i.e., a posterior predictive plot) showing that the model captured the data well. 


\section{Appendix B}

Bayesian Model Predicting JOLs by Sequence-Length and Test Condition We explored whether JOLs are influenced by the test condition and sequence-length by estimating a Bayesian model in brms. To model participants' JOLs, we used the zero-or-one inflated beta regression (ZOIB), a method to analyze data from continuous response scales. The main assumption of ZOIB is that the observed distribution of scale-response data evolves from two underlying distributions: A binomial distribution (participants either choose the highest or the lowest value of the scale) and a beta distribution (participants choose values in the whole range of the scale). The parameter phi caputers the precision of the beta distribution, the parameter zoi reflects the probability to be in the binomial distribution and the parameter coi reflects the proportion of responses in the upper end of the scale. In our model, we predicted average JOLs as well as all three free parameter by sequence-length, test condition and their interaction. To apply this model, we first linearly transformed the JOLs into a scale ranging from 0 to 1 . The variable sequence-length was again centered on length 2 . We fitted the model with 4 chains, each with 10000 iterations (5000 iterations were discarded as burn-in). Parameters converged as indicated by all R-hat values being below 1.05 .

Table B1 depicts the model's population level estimates of the effects of sequence-length, test condition and the interaction of sequence-length and test condition on average JOLs (see Supplementary Materials File for a comprehensive overview of effects in all parameters). The ZOIB results replicated the findings from the Bayesian ANOVA: There was an interaction of sequence-length and test time-point on JOL and likewise two main effects of test time-point and sequence-length on JOLs. 


\section{Table B1}

Population-Level Estimates for the Effects of Sequence-Length, Test Time Point, and Their Interaction on JOLs. Values are Reported on a Logit Scale.

\begin{tabular}{lcccc}
\hline & $\begin{array}{c}\text { Posterior } \\
\text { Estimate }\end{array}$ & $\begin{array}{c}\text { Posterior } \\
\text { Estimate } \\
\text { Error }\end{array}$ & $\begin{array}{c}\text { lower 95\% } \\
\text { CI }\end{array}$ & $\begin{array}{c}\text { upper 95\% } \\
\text { CI }\end{array}$ \\
\hline Sequence Length & $\mathbf{- 0 . 0 8}$ & $\mathbf{0 . 0 2}$ & $\mathbf{- 0 . 1 2}$ & $\mathbf{- 0 . 0 3}$ \\
Test Time Point & $\mathbf{0 . 7 8}$ & $\mathbf{0 . 2 9}$ & $\mathbf{0 . 2 2}$ & $\mathbf{1 . 3 7}$ \\
$\begin{array}{l}\text { Sequence } \\
\text { Length*Test Time } \\
\text { Point }\end{array}$ & $\mathbf{- 0 . 1 2}$ & $\mathbf{0 . 0 3}$ & $\mathbf{- 0 . 1 8}$ & $\mathbf{- 0 . 0 6}$ \\
\hline Note. CI = credible interval. Credible parameter values (i.e., for which 0 is not \\
included in the credible interval) are presented in bold.
\end{tabular}




\section{Appendix C}

Bayesian Model Predicting Test Performance (Kappa) in Delayed Test by SequenceLength and Recall Error in Immediate Test

We estimated a Bayesian mixed-effects model in brms with immediate recall error and sequencelength as two continuous predictors and delayed recall error as dependent variable. Statistically, this prediction should be reflected in the interaction between sequence-length and immediate recall error on delayed recall error. Instead of predicting the recall error directly, we again used the von Mises distribution and modeled the precision parameter, Kappa. We fitted the model with 4 chains, each with 2000 iterations (1000 iterations were discarded as burn-in). Parameters converged as indicated by all R-hat values being below 1.05. Figure $\mathrm{C} 1$ depicts the models' predictions.

\section{Figure C1}

Prediction for Kappa (Memory Precision) as a Function of Sequence-Length and Immediate Recall Error for the Data of Experiment 2.

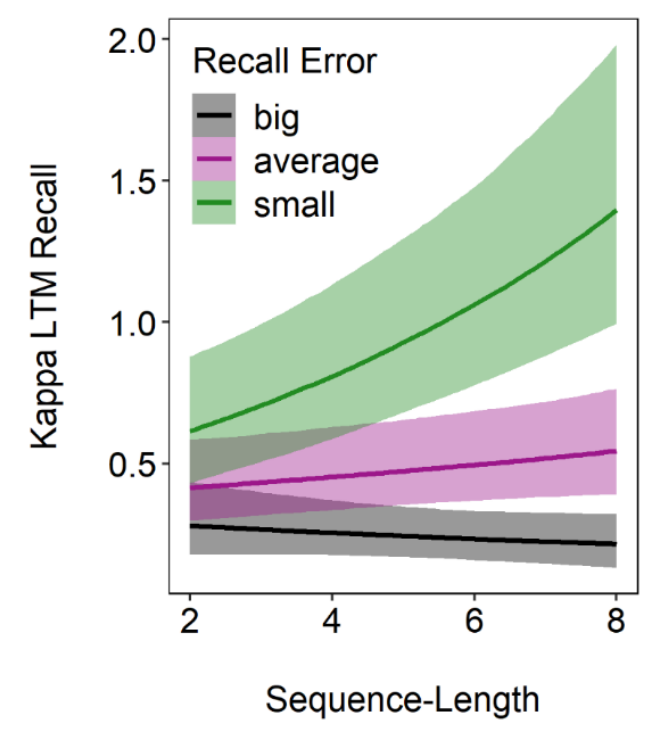

Note $. \mathrm{Big}=$ recall error that is one standard deviation above the average recall. Small $=$ recall error that is one standard deviation below the average. 
As expected, the model predicts an interaction between immediate recall error and sequence-length on the precision of recall in the LTM test (see Table C1 and Figure C1). At a sequence-length eight, the immediate recall error is more predictive for the delayed recall Kappa than at a sequence-length two. In other words, when participants perform well in the immediate test in the sequence-length eight condition, they are more likely to perform well on that object in the delayed test. In contrast, performing well on an object in the immediate test in a sequencelength two condition is weakly associated with better performance in the delayed test. Thus, the modelling result confirms our prediction: immediate and delayed recall error are higher correlated in the sequence-length eight condition.

\section{Table C1}

Population-Level Estimates for the Effects of Sequence-Length, Immediate Recall Error, and Their Interaction on Memory Precision (Kappa) in the Delayed Test. Values are reported on a logarithmic scale.

\begin{tabular}{lcccc}
\hline & $\begin{array}{c}\text { Posterior } \\
\text { Estimate }\end{array}$ & $\begin{array}{c}\text { Posterior } \\
\text { Estimate } \\
\text { Error }\end{array}$ & $\begin{array}{c}\text { lower 95\% } \\
\text { CI }\end{array}$ & $\begin{array}{c}\text { upper 95\% } \\
\text { CI }\end{array}$ \\
\hline Sequence Length & $\mathbf{0 . 1 2}$ & $\mathbf{0 . 0 2}$ & $\mathbf{0 . 0 8}$ & $\mathbf{0 . 1 6}$ \\
$\begin{array}{l}\text { Immediate Recall } \\
\text { Error }\end{array}$ & $\mathbf{- 0 . 5 8}$ & $\mathbf{0 . 1 6}$ & $\mathbf{- 0 . 9 3}$ & $\mathbf{- 0 . 2 8}$ \\
$\begin{array}{l}\text { Sequence } \\
\text { Length* } \\
\text { Immediate Recall } \\
\text { Error }\end{array}$ & $\mathbf{- 0 . 1 3}$ & $\mathbf{0 . 0 4}$ & $\mathbf{- 0 . 2 1}$ & $\mathbf{- 0 . 0 5}$ \\
\hline Note. CI = credible interval. Credible parameter values (i.e., for which 0 is not \\
\\
included in the credible interval) are presented in bold.
\end{tabular}

